\title{
A not so random walk through space, time and spirit
}

\author{
Barry J. Allen ${ }^{1}$
}

Published online: 2 November 2015

(C) Australasian College of Physical Scientists and Engineers in Medicine 2015

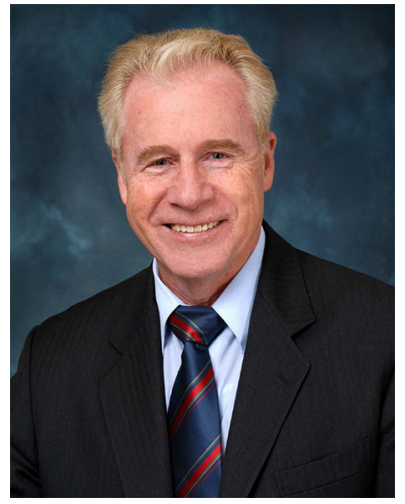

I was appointed an Officer in the Order of Australia award in the Queens birthday honours list for 2015. The citation read "for distinguished service to biomedical physics, particularly to radiation oncology and the development of innovative methods of cancer treatment, and to international professional scientific organisations".

My research experience ranges from the formation of radioisotopes in stars to their use in cancer therapy. Although having only two long term appointments, I was fortunate to have the freedom to allow my self-direction and motivation to develop my research interests.

After graduating MSc in nuclear physics at the University of Melbourne, I joined the Australian Atomic Energy Commission (AAEC) at Lucas Heights in 1963. The AAEC was expanding rapidly, developing nuclear based research projects for reactors and accelerators. I began my R\&D using the $3 \mathrm{MV}$ Van de Graaff accelerator

\footnotetext{
Barry J. Allen

bja1940@optusnet.com.au

1 Yowie Bay, NSW, Australia
}

to produce neutron beams at $\mathrm{keV}$ energies for neutron capture gamma ray spectroscopy. Neutron energy was measured by pulsed beam time of flight and gamma ray energy by world first $\mathrm{Ge}(\mathrm{Li})$ high resolution gamma spectrometry in three dimensional on-line computer studies. The $\mathrm{keV}$ neutron capture gamma ray project was to provide data for fast reactor calculations and to investigate the underlying mechanisms for potential and resonance neutron capture.

Man landed on the moon, I married Cynthia and together we landed in Atomic City, Oak Ridge, Tennessee, USA on attachment to the Oak Ridge National Laboratory (ORNL) in 1969. My job was to study high resolution resonance neutron capture reactions in collaboration with Dr. Dick Macklin. The world leading $140 \mathrm{MeV}$ Linear Accelerator (ORELA) was used as a high resolution, pulsed source of time of flight neutrons for a wide range of capture studies on separated isotopes. These studies continued with a later posting to Central Bureau for Nuclear Measurements (CBNM) in Belgium, with the additional task of multilingual communication and extensive vacation. Some 105 collaborative papers were published in these fields.

This research was also relevant to the slow neutron capture process of stellar nucleosynthesis, which occurs at a Maxwellian neutron temperature of $\mathrm{kT}=30 \mathrm{keV}$ and governs the creation of most of the heavier isotopes across the periodic table (Fig. 1).

A highlight of the neutron research program was attending in 1982, the 50th anniversary conference of the discovery of the neutron at Cambridge. The worn sandstone steps and the Bridge of Sighs over the river Cam reflected the long history of scientific excellence. However, it was clear that neutrons were sliding down the totem pole at the AAEC and new directions were needed. 
Stellar Nucleosynthesis at $30 \mathrm{keV}$

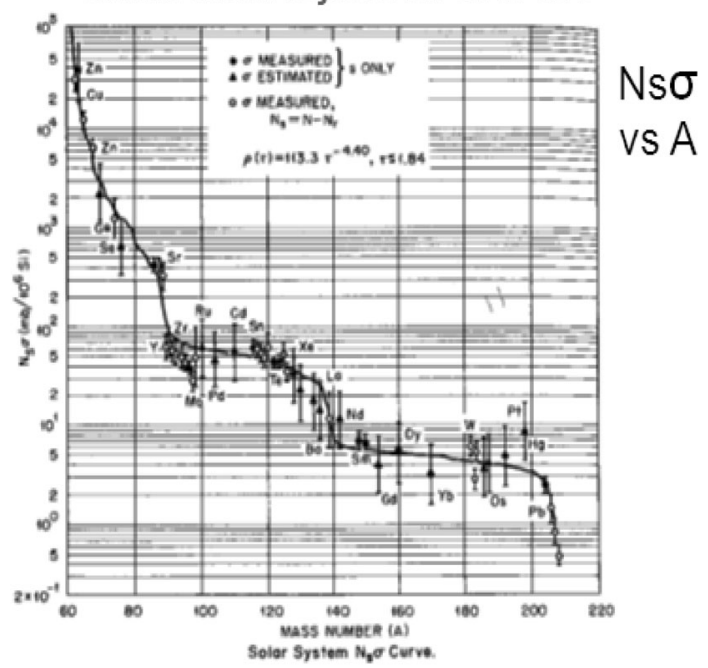

Fig. 1 The product of s-process abundances \& $30 \mathrm{keV}$ neutron capture cross sections $\left(\mathrm{N}_{\mathrm{s}} \sigma\right)$ vs mass number over 5 orders of magnitude, shows that "the leaves of grass are writ in the stars".(Walt Whitman)

\section{Development of innovative methods of cancer treatment}

I began neutron based, medical $\mathrm{R} \& \mathrm{D}$ programs in Boron Neutron Capture Therapy (BNCT) for cancer and In Vivo Body Composition (IVBC) for medicine.

I designed the in vivo nude mouse irradiation facility at the Moata reactor at Lucas Heights, demonstrating for the first time the induction of double strand breaks in DNA arising from neutron capture induced auger emission (with Dr. Roger Martin, Peter MacCallum Cancer Centre). Among other firsts were the boron assays in biological tissues by inductively coupled plasma atomic emission spectrometry and energy loss spectroscopy with an electron microscope (with Dr. Doug Moore, University of Sydney).

The role of heavy water in Boron Neutron Capture Therapy to extend the treatment depth for thermal neutrons was fully explored. The uptake of heavy water and its biological effect were measured in clinical studies in Japan (with Ned Blagojevic \& Prof. Hiroshi Hatanaka).

Monte Carlo calculations were made to determine the microscopic impact of the high linear energy transfer particles; with particular reference to ion passages through brain endothelial nuclei during boron neutron capture therapy (with Dr. David Charlton).

Four annual BNCT workshops for melanoma were held alternately in Kobe and in Sydney (with Prof. Yutaka Mishima). I went on to become President of the International Society for Neutron Capture Therapy and to convene the Fourth International Symposium for Neutron Capture Therapy in Sydney in 1990.
The collaborative research produced 87 refereed conference and journal publications on all aspects of BNCT, ranging from the physics of radiation dosimetry, boron measurement and reactor and accelerator neutron beams, chemistry of boron chemicals, proteins, monoclonal antibodies and liposomes, the radiobiology of high linear energy transfer reactions and radiation oncology for melanoma and glioblastoma.

The Australian NCT effort came to a halt in 1994 with the closure of the Moata reactor and the lack of recognition by the Australian Nuclear Science and Technology Organisation (ANSTO) of the strategic importance of installing an epithermal neutron beam facility in the new replacement reactor. An epithermal beam was not even considered by the Neutron Beam Committee, showing that bad science can always win the day. However, the NCT project with its multidisciplinary structure was a great training ground for me. This was especially important for the later development of targeted alpha therapy (TAT) at St George Hospital.

In the 1980's, I designed the first human Body Protein Monitor (BPM) in Australia, which was fabricated by ANSTO at Lucas Heights (with Ned Blagojevic). Initially for paediatric studies of protein change in cystic fibrosis patients (with Dr. Kevin Gaskin), the BPM was later installed at Royal North Shore Hospital (with Prof. Ross Smith) for collaborative clinical studies with most Sydney hospitals. Prominent research areas were renal failure, AIDS, cancer and surgery. I installed a second BPM at the University of Texas Medical Branch in Galveston. A third unit was installed at the Westmead Children's Hospital (Fig. 2) Some 55 refereed papers were published in collaboration with researchers in clinical studies in these specialist fields.

I commenced the Targeted Alpha Therapy project in 1994 at St George Hospital, soon to be joined by Dr. Syed Rizvi (radiochemistry) and Dr. Chand Raja (data analysis). This project was successful in developing new preclinical agents for the treatment of melanoma, leukaemia, breast, prostate, pancreatic, ovarian and colorectal cancers.

I was the designer and Study Director of two world first trials of intralesional and systemic targeted alpha therapy for metastatic melanoma. 51 patients were treated in these Phase 1 dose escalation trials (with Dr. Peter Graham, Medical Director).

To date, there have been 72 publications in TAT. The clinical trial results were my most important papers and gave rise to the tumour anti-vascular alpha therapy (TAVAT) concept for the regression of solid tumours by alpha therapy. I am rather proud of this paper as I regard it as one of my best and most important, but perhaps the least read. It explains how the short range alpha radiation emitted by the radio-immunoconjugate can regress solid 


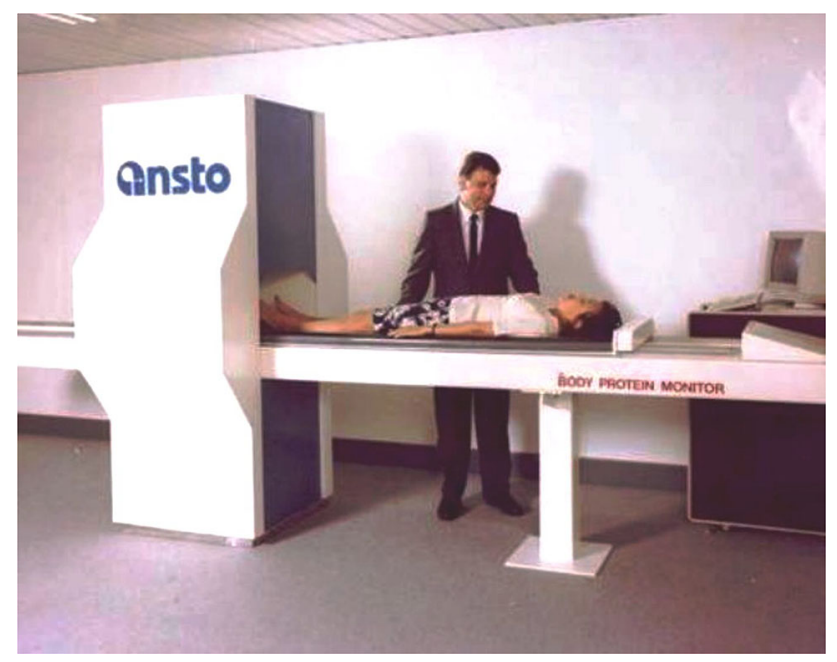

Fig. 2 ANSTO installed a BPM at the Westmead Children's Hospital as an important research tool in studying the efficacy of management of many paediatric diseases and treatments, especially cystic fibrosis and renal disease

tumours by targeting perivascular cancer cells. Alpha radiation from these cells creates a biological alpha field along the capillary axis, killing the endothelial cells and closing up the capillary to inhibit tumour growth. The TAVAT concept has been validated by micro Monte Carlo calculations (with Dr. Chen-Yu Huang)

High linear energy transfer dosimetry is required for clinical TAT. A further important development was the first demonstration of clinical biological dosimetry for systemic alpha therapy (with Dr. Emma Song), which was based on the formation of micronuclei in lymphocytes randomly hit by alpha emissions in the blood.

On behalf of the Australasian College of Physical Scientists \& Engineers in Medicine, I led the successful bid for the 2003 World Congress on Medical Physics and Biomedical Engineering, to be held in Sydney (Fig. 3). Although preceded by the $9 / 11$ terrorist attack and beset by the outbreak of the infectious disease SARS, the Congress achieved its objectives in Sydney with some 2200 delegates attending on a $\$ 2000,000$ budget. The Congress Committee was able to exercise the essential fiscal restraint to achieve a satisfactory profit for the congress.

I have published over 340 papers in neutron and biomedical physics. Research topics included neutron capture gamma rays, resonance cross sections, stellar nucleosynthesis, clinical in vivo body composition, neutron capture therapy, macro and micro-dosimetry, micro-radiation beams, biological dosimetry, preclinical and clinical targeted alpha therapy and appropriate technology for developing countries. I co-authored the text book "Biomedical Physics in Radiotherapy for Cancer" published in 2011 with Drs. Eva Bezak and Loredana Marcu.

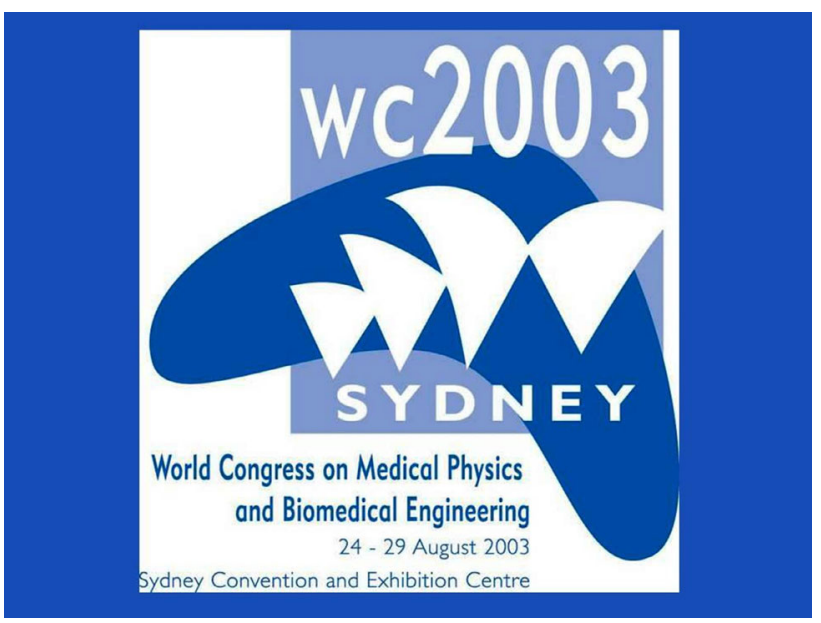

Fig. 3 Logo for the 2003 world congress in Darling Harbour, Sydney

Conjoint Professorships were held at the Universities of Sydney, NSW, Wollongong and Western Sydney. Previously, I worked at the AAEC as an Experimental Officer 1, rising to Chief Research Scientist to the chagrin of Management. More recently I was a Principal Medical Physicist Specialist in the Cancer Care Centre and Clinical School at St George Hospital in Sydney.

I was awarded Fellowships by the Australian Institute of Physics (1972), the American Physical Society (1981), the Australasian College Physical Scientists \& Engineers in Medicine (1992) and the Institute of Physics (1999).

\section{Contributions to international professional scientific organisations}

I was elected President of the International Society for Neutron Capture Therapy in 1988, the Australasian College of Physical Scientists \& Engineers in Medicine in 1998, the Asia-Pacific Federation of Medical Physics (AFOMP) in 2003, the International Organisation for Medical Physics in 2006 (IOMP) and the International Union of Physical \& Engineering Sciences in Medicine (IUPESM) in 2009.

I introduced many new initiatives in these organisations. With regard to AFOMP, I further developed the AFOMP logo (with Dr. Kiyo Inamura) to include the Chinese characters, taken from a Japanese temple, that spiritualize the essential elements of our discipline in the Asian context, viz: cosmos 宙, time 時 and spirit 魂. As the majority of AFOMP members have Chinese as a first or second language, it made sense to acknowledge this fact in the logo.

I facilitated the formation of the Vietnamese Association for Medical Physics, which hosted the 2008 AFOMP 
meeting, together with my Workshop on Palliative Radiotherapy for Developing Countries.

In keeping with furthering the IOMP policy of regional groups, I initiated the establishment of the Middle East Federation of Medical Physics (MEFOMP) and proposed the first regional conference, which was held in Shiraz, Iran.

With regard to the IOMP, I developed a new look Medical Physics World (with Dr. Ishmael Parsai), putting more emphasis and colour on scientific achievements and less on the organisation itself.

I established the Health Technology Task Group (HTTG) of the IUPESM to assist developing countries in the implementation of appropriate technology and was the inaugural Chair.

\section{Space, time and spirit}

The 50th Anniversary Conference on Medical Physics was held at Brighton, UK, September 2013. I was nominated by our College and accepted for inclusion in the 50 outstanding medical physicists over the past 50 years.

I have indeed been fortunate in being in the right place at the right time. Unemployable in Melbourne with an MSc degree in nuclear physics in 1973, the AAEC had recently been established with its research centre at Lucas Heights and was in dire need for nuclear physicists. Years later, that need had abated but I was able to start national and international research programs on the application of neutrons in medicine. However, by 1994, bureaucracy had trumped initiative at ANSTO, the renamed AAEC. I moved to an R\&D position in radiation oncology at St George Hospital (with Dr. John Kearsley), where the targeted alpha therapy project was developed from scratch with research funds from successful competitive grants, mostly with the US Department of Defence.

I put together a multidisciplinary research team that developed a sound basis for TAT by first in vitro and then in vivo studies for a range of cancers and targeting vectors. These studies led to the intralesional and systemic phase 1 clinical trials for metastatic melanoma. But laboratory and publication successes could not save the TAT project in the face of the fierce determination of hospital management to cut costs, regardless of the government in power. In the end, the NSW Department of Health was responsible for disrupting and shutting down the preclinical and clinical research of its own cancer scientists, first at Prince of Wales Hospital and then St George Hospital. To do so in the middle of a world first clinical trial of TAT for metastatic melanoma was unconscionable behaviour.

Our College was successful in nominating me for an Order of Australia award, being appointed an Officer of the Order (AO). Such an honor should be shared by the College, as we all contribute to our individual successes. It is also important to emphasize the multidisciplinary collaborations at the national and international levels and the essential contribution of many Ph.D. students and colleagues to my $R \& D$ projects.

While the reader might see an unbroken career of successful research that brings together space, time and spirit, the reality is rather different. With space, I have been fortunate to work in Australia and overseas in top research centers. With time, my research has been designed to answer current and future needs. However, the spirit has been sorely tested by management on several occasions but has been supported by independent reviewers. The struggle for grant funding is also an ongoing trial and challenges your belief in the need for your research. This means that self-critique, as distinct from delusion, is an essential element in the research process.

Medical Physics is a demanding discipline and while our internal clinical standards continue to improve, we are failing to obtain adequate $R \& D$ time under the award to support the development of further research applications. This should be an essential role for our academic members, who can also collaborate with clinical medical physicists to further expand the biomedical applications of physics.

Research funding is highly competitive and dominates the survival of new ideas and the careers of researchers. My major research opportunities were few but I was funded by my employers. However, such research positions may not be available for our next generation of scientists, so young researchers should make sure that they have a good day job as well.

Finally, in my involuntary retirement, I wish my younger colleagues good fortune in their endeavors to serve our patients and restore their health. However, I ask you to remember that while external beam is an essential therapeutic modality, systemic disease is the ultimate task that still awaits a therapeutic solution for many end-stage cancers. 